\title{
Narrativa
}

\section{Robledo o la "bufala" dei ghost class heroes: investigare la precarietà attraverso la falsificazione}

\section{Monica Jansen}

\section{(2) OpenEdition}

\section{Journals}

\section{Edizione digitale}

URL: https://journals.openedition.org/narrativa/334

DOI: $10.4000 /$ narrativa.334

ISSN: 2804-1224

\section{Editore}

Presses universitaires de Paris Nanterre

\section{Edizione cartacea}

Data di pubblicazione: 1 décembre 2020

Paginazione: 165-178

ISBN: 978-2-84016-381-7

ISSN: $1166-3243$

Notizia bibliografica digitale

Monica Jansen, «Robledo o la "bufala" dei ghost class heroes: investigare la precarietà attraverso la falsificazione», Narrativa [Online], 42 | 2020, online dal 01 décembre 2021, consultato il 08 décembre 2021. URL: http://journals.openedition.org/narrativa/334 ; DOI: https://doi.org/10.4000/narrativa.334

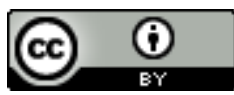

Narrativa est mise à disposition selon les termes de la Licence Creative Commons Attribution 4.0 International. 


\section{Robledo o la "bufala" dei ghost class heroes: investigare la precarietà attraverso la falsificazione}

\section{RIASSUNTO}

Il romanzo Robledo di Daniele Zito è costruito come un'edizione postuma dei "quaderni" del giornalista Michele Robledo che raccolgono la sua investigazione sull'organizzazione clandestina del Lavoro Per il Lavoro (LPL) i cui membri compiono il loro "percorso di liberazione" suicidandosi sul posto di lavoro. Secondo Daniele Zito, autore della "Postfazione", Robledo ha condotto l'indagine sul lavoro non salariato attraverso la "falsificazione". In questo modo, con una narrazione che combina sincerità e finzione speculativa, Zito riesce a raccontare sia il precario come "antieroe" dei nostri tempi sia a riprodurre, attraverso la "distorsione" della "bufala", l'elemento umano della verità sociale.

\section{RÉSUMÉ}

Le roman Robledo de Daniele Zito est construit comme une édition posthume des "cahiers" du journaliste Michele Robledo qui rassemblent son enquête sur l'organisation clandestine Lavoro Per il Lavoro (LPL - Travail Pour le Travail) dont les membres achèvent leurs "parcours de libération" en se suicidant sur leur lieu de travail. D'après Zito, auteur de la Postface, Robledo a mené son enquête sur le travail non rémunéré à travers la "falsification". De cette façon, par une narration qui conjugue sincérité et fiction spéculative, Zito parvient aussi bien à raconter le travailleur précaire comme un "antihéros" de notre époque qu'à reproduire, par la "distorsion" du canular, l'élément humain de la vérité sociale. 


\section{LA “FOLLIA Plausibile” COME CHIAVE Di LETTURA DELLA REALTÀ}

Robledo esce per Fazi nel 2017 e in traduzione francese, per Christian Bourgois, nel $2019^{1}$. Daniele Zito, nato a Siracusa nel 1980 e residente a Catania, ha a suo nome già un romanzo, La solitudine di un riporto ${ }^{2}$, salutato con entusiasmo sul Sole 24 Ore da Andrea Bajani ${ }^{3}$, il cui invito alla lettura di Robledo campeggia in copertina: "Leggete Bolaño e Foster Wallace e poi dimenticateli. Si rifaranno vivi loro buttando giù la porta delle pagine di Zito". I due scrittori suggeriti da Bajani segnano la svolta internazionale verso nuovi realismi dopo il postmoderno: il primo è considerato da Donnarumma come esempio di un autore "ipermoderno", il secondo viene identificato dalla critica internazionale come un antesignano della cosiddetta "nuova sincerità" in cerca di una nozione di verità dopo l'ironia postmoderna 5 .

Secondo la finzione del manoscritto ritrovato, Robledo ricostruisce post mortem $\mathrm{i}$ "quaderni” del giornalista Michele Robledo. L'edizione dell'opera è curata da un certo A.B., con il sostegno della Fondazione Robledo, e corredata da una "Postfazione" di Daniele Zito. Robledo sarebbe stato il primo a far emergere la verità dell'organizzazione clandestina del Lavoro per il Lavoro (LPL) che, secondo la cronaca riportata da Zito nella "Postfazione", in "quasi quattro anni d'attività [...] ha provocato la morte cruenta di centinaia di lavoratori non convenzionali", Robledo stesso, identificato dalle forze dell'ordine come il "cattivo maestro" del movimento, finisce prima in carcere, poi in una clinica psichiatrica e alla fine muore compiendo il suo "percorso di liberazione" lasciando un "biglietto d'addio" come hanno fatto i protagonisti delle sue inchieste. Il biglietto di Robledo, sempre secondo lo Zito della "Postfazione", sarebbe l'unico documento sulla cui

1. Zito Daniele, Robledo, Roma, Fazi, 2017; Robledo, tr. fr. di Lise Chapuis, Paris, Christian Bourgois, 2019.

2. Zito Daniele, La solitudine di un riporto, Matelica, Hacca edizioni, 2013.

3. Bajani è un autore che appartiene al "canone" delle narrazioni sul lavoro. Si pensi alla sua indagine sui lavoratori precari Mi spezzo ma non m’impiego. Guida di viaggio per lavoratori flessibili, Torino, Einaudi, 2006, e ai romanzi Cordiali saluti, Torino, Einaudi, 2005 e Se consideri le colpe, Torino, Einaudi, 2007.

4. Donnarumma Raffaele, "La macchina dell'oscurità", Allegoria 71-72, 2015, p. 226: "Siamo ormai in un clima ipermoderno: i debiti con il passato recente ci sono, ma si respira un'altra aria. E siamo, anzitutto, in piena riabilitazione del romanzo come invenzione di mondi che hanno a che fare con la nostra esperienza del quotidiano".

5. Si veda p.es. Kelly Adam, "David Foster Wallace and the New Sincerity in American Fiction", in Hering David (a cura di), Consider David Foster Wallace, Sideshow Media Group, 2010, pp. 131-46.

6. Ziтo Daniele, Robledo, cit., p. 257. 
veridicità non affiorano dubbi, mentre tutto ciò che ha scritto nei suoi undici quaderni sarebbe invece, secondo la sua opinione, "falso"”.

Il curatore A.B. nella sua premessa rileva come nella presente edizione siano stati inclusi due quaderni, "Diario I" e "La prima pietra", che invece secondo molti studiosi costituirebbero degli apocrifi. A.B. così facendo ha voluto "rendere conto del fitto dibattito attorno alla paternità delle opere roblediane" Zito, nella "Postfazione", inoltre, osserva che proprio questi testi di Robledo "rappresentano l'apice della sua arte" e che "sono le chiavi con le quali Robledo ha provato a scardinare la credenza comune che i fatti possano essere registrati in maniera obiettiva, senza sbavature o tensioni, nella loro nudità, dimostrando che sono le parole a creare i fatti, e non viceversa"'.

Uno dei tratti più originali del romanzo è infatti la cura pseudofilologica con cui Zito ha costruito i paratesti dei quaderni roblediani, inclusi il "Piano originario dell'opera" in appendice, l'apparato delle note e i "riferimenti bibliografici", il tutto per rendere ancora più "plausibile" la veridicità dei quaderni. L'evidente gusto del gioco letterario si palesa in titoli di studi critici quali: "Morana G., Apocrifi e integrati, Laterzia, 2014" e "Morana G., Palummo P., Spicuglia C., Apocrifi e disintegrati, Laterzia, 2015"10, con un'evidente strizzata d'occhio al famoso saggio di Umberto Eco, Apocalittici e integrati, e alle varianti sul suo titolo nei diversi contributi al dibattito sul postmoderno in Italia ${ }^{11}$.

Secondo A.B. neanche questa volta si è potuto includere il reportage Ghost Class Heroes, per "ragioni di copyright"12. Per questo motivo le osservazioni metatestuali di Robledo nel "Diario I" sulla genesi del suo primo reportage che ha causato tante polemiche sarebbero da intendere come "chiavi", come suggerisce Zito nella "Postfazione" sopra citata. Robledo è in cerca di un'idea vincente che gli permetterà di "risalire la china"13: sarà una commessa in un negozio di vestiti a dargli "l'idea della svolta" ${ }^{14}$. La immagina "un'impostora" che si spaccia per commessa, nello stesso modo in cui lui stesso è un "infiltrato", che da dieci anni fa il giornalista senza aspettarsi "la benché minima forma di

7. Ibid., p. 255.

8. Ibid., p. 14.

9. Ibid., p. 259.

10. Ibid., p. 265.

11. Eco Umberto, Apocalittici e integrati, Milano, Bompiani, 1964. Cfr. anche Lorusso Anna Maria (a cura di), 50 anni dopo apocalittici e integrati, Roma, DeriveApprodi, 2015.

12. Ziтo Daniele, Robledo, cit., p. 15.

13. Ibid., p. 39.

14. Ibid., p. 46. 
retribuzione ${ }^{15}$. La sua indagine parte da ciò che lui chiama una "follia plausibile" (corsivo nell'originale) o da una "congettura", come in un "romanzo o un film di fantascienza"16. Ipotizza che "la chiave" di questa condizione comune a "centinaia, migliaia, milioni di persone" sia il "lavorare per il gusto di lavorare", perché "siamo schiavi felici di esserlo" 17 . Da semplice idea, il lavoro per il lavoro diventa per il giornalista precario "un'ossessione", espandendosi fino a diventare "un fenomeno che si autoalimenta grazie alla sommatoria di migliaia di micro-fenomeni individuali che intrecciandosi diventano un'emergenza collettiva" ${ }^{\prime 1}$. Inoltre, il giornalista sente il bisogno di scriverne essendo quella la sua "unica possibilità di pubblicare"19. Così impiega tutte le sue energie per "mettere assieme i pezzi di un reportage inventato" su questi lavoratori non convenzionali che chiamerà Ghost Class Heroes richiamandosi al titolo della canzone Working Class Hero di John Lennon aggiungendo di non essere sicuro di averlo "storpiato nella maniera corretta": "Devo riuscire a dargli un corpo e una postura. Devo riempirlo di storie. Devo renderlo plausibile" ${ }^{20}$, scrive il giornalista quarantenne sul suo diario, in preda alla sua follia mentre sta per essere lasciato da Veronica, la sua fidanzata di quindici anni più giovane.

Nel "Diario I", Robledo espone quindi la genesi del suo futuro capolavoro soffermandosi anche sui risvolti amorali ${ }^{21}$ della sua impresa: servendosi di dati statistici reali relativi ai "morti sul lavoro", il giornalista riesce a dare plausibilità all'ipotesi della dipendenza dal lavoro che spinge i ghost worker a suicidarsi. Agisce "nell'ombra", selezionando fra i morti sul lavoro "i casi più discussi" che non possono "smentire" la sua versione dei fatti, e ci costruisce attorno "un teorema". Dice che la riuscita del suo capolavoro dipenderà però anche dalla collaborazione dei lettori, per cui decide di "puntare tutto sull'immedesimazione"22. Il "Diario I" offre quindi tutti gli elementi per far concludere a Zito, nella sua "Postfazione", che Robledo "investigava la realtà attraverso la

15. Ibid., pp. 47-48.

16. Ibid., p. 48.

17. Ibid., p. 50 e p. 49.

18. Ibid., pp. 51-52.

19. Ibid., p. 52 .

20. Ibid., p. 55 e p. 53.

21. "Quello che sto facendo è orribile. Uso la cronaca per raschiare il fondo e, invece di allontanarmi inorridito, aguzzo le antenne, rimesto nel fango, creo" (Ibid., p. 59).

22. Ibid., p. 60. 
falsificazione"23. Invece "La prima pietra (Quaderno X)", l'ultimo reportage scritto da Robledo in clinica, esprimerebbe, sempre secondo Zito, l'incredibile forza del giornalista di compiere l'ultimo passo del "parlare di sé, raccontare la propria esistenza, abbandonare ogni idea di ribellione alla verità, accettare la sincerità come unica forma di conoscenza" ${ }^{24}$.

È qui che Bolaño e Foster Wallace "buttano giù la porta delle pagine di Zito": falsificazione letteraria e sincerità non si contraddicono, ma i due quaderni apocrifi, secondo Zito, sono "autentici" senza pertanto essere "veritieri" 25. Nell'universo della narrazione, accade che molti lettori si riconoscano nella "voce rauca di un barbone" di Robledo che "conteneva di tutto"26, tanto che, secondo i dati forniti da A.B., il libro in sette mesi "è già giunto alla sesta ristampa"27. Il curatore riconosce il merito dei quaderni proprio nell'incessante intrecciarsi di "verità storica e verità romanzesca", il che avrebbe indotto gli studiosi, intenti a screditarne l'autenticità, a parlare di "postverità" 28 . Secondo il curatore "il cuore della questione" - e qui cita le parole di Robledo stesso dal mancante terzo quaderno Ghost Class Heroes - sarebbe proprio il fatto che "Ogni versione è ugualmente plausibile. Tutte, però, possono essere confutate, e nessuna smentita del tutto. A chi credere? Di chi diffidare?"29.

Zito nella "Postfazione" sostiene che "reportage e movimento politico sono talmente intrecciati tra loro" che assurdamente "si potrebbe affermare che sono venuti alla luce assieme, nello stesso identico momento, l'uno l'immagine speculare dell'altro $^{30}$. Il curatore A.B. invita i lettori a intraprendere i propri percorsi di lettura, a lasciarsi trascinare dalla prosa dei quaderni e leggerli come un "puro oggetto letterario", a "confrontare le proprie conoscenze con quelle di Robledo in modo da illuminare meglio vicende e personaggi che appartengono alla storia di questi anni", a "tentare di dipanare il mistero di un autore tanto singolare

23. Ibid., p. 256.

24. Ibid., p. 257.

25. Ibid., p. 258.

26. Ibid., p. 259

27. Ibid., p. 12.

28. Si vedano nei "Riferimenti bibliografici" gli studi (inventati) di Martorana M., Marxismo vs Post-Verità: il caso Robledo, Alias Ed, 2014, A. Scalia, Post Verità e Menzogna in Robledo, AlterAlter, 2011 (Ibid., pp. 265- 266). Il riferimento alla postverità permette un altro rimando a Eco, al suo ultimo romanzo: Eco Umberto, Numero zero, Milano, Bompiani, 2015 (cfr. De Benedictis Raffaele, "Lying, a Way of Knowledge in Umberto Eco's Numero Zero", in Forum Italicum, 51, 1, 2017, pp. 170-186).

29. Ziтo Daniele, Robledo, cit., p. 13 (corsivo nell'originale).

30. Ibid., p. 256. 
quanto sfuggente" ${ }^{31}$. Seguiamo il secondo suggerimento per analizzare come Zito riesca a ponderare la plausibilità di ogni versione del reale attraverso la finzione del reportage.

\section{IL PRECARIO, ANTIEROE DEI NOSTRI TEMPI}

Le recensioni dell'esordio insieme alle interviste all'autore offrono dei contesti sociali e culturali per verificare se, come dichiara il curatore nel romanzo, "leggere un reportage di Robledo vuol dire penetrare nel cuore oscuro della società" ${ }^{\prime 2}$. Un primo indizio può essere la biografia di Zito con cui veniva presentato ai lettori dell'esordio La solitudine di un riporto:

In questi ultimi tempi i ricercatori precari sembrano destinati a fare tutt'altro. Nel film Smetto quando voglio [...] si danno al traffico di pasticche per sbarcare il lunario. Daniele Zito, invece, oltre a occuparsi - come da biografia - di "cloud e sistemi complessi tempo varianti" per l'Università di Catania, si dà alla scrittura"333.

Il riferimento alla commedia di grande successo di Sydney Sibilia, inclusa in una trilogia, permette di stabilire un nesso con il fenomeno delle rappresentazioni cinematografiche del precariato accademico da cui emerge la figura del "giovane ricercatore", con un'età media di 35 anni, relegato a posizioni talmente marginali che riesce difficile definirne la posizione. Questi lavoratori atipici vivono in balìa di sentimenti di "anomia" (passività e disperazione) $)^{34} \mathrm{e}$ frustrazione di status dovuti alla negazione di sbocchi futuri. La loro trasformazione in "microimprenditori" che, soli ma interconnessi, lavorano sempre di più a scapito delle relazioni sentimentali e procreative, fa aumentare la loro rabbia spingendoli ad atti vendicativi che nella chiave tragicomica di questi film di genere porta il pubblico a riflettere criticamente sull'assurdità di questa "normalità" alienante nascosta sotto l'etichetta di "early-career researchers" 35 .

31. Ibid., p. 13.

32. Ibid., p. 12.

33. Aloise Salvatore, L'Internazionale, 14 Marzo 2014, riportato nel blog di Zito Sei cose: http://seicose.blogspot.com/p/recensioni-interviste-rocchenroll.html

34. Il riferimento è a STAnding Guy, The Precariat. The New Dangerous Class opera seminale in cui sostiene che "The precariat experiences the four A's - anger, anomie, anxiety and alienation” (London, Bloomsbury Academic, 2011, p. 19). Cit. da BARACCO Alberto, "I can quit whenever I want. The academic precariat in Italian cinema", in Toracca Tiziano e Condello Angela (a cura di), Law, Labour and the Humanities, London, Routledge, 2020, p. 203.

35. Ibid. 
Come spiega Zito nelle varie interviste rilasciate dopo la presentazione di Robledo al Salone del Libro di Torino, punto di partenza per la sua finzione sui ghost workers sono stati gli amici nell'ambiente universitario di Catania che, come l'autore stesso, accettavano di fare lavori non retribuiti con la promessa di strutturarsi in un futuro prossimo facendosi la "gavetta":

Robledo nasce da una domanda ingenua [...]: Perché accettiamo di lavorare senza essere pagati? Cosa ci spinge a farlo? Può sembrare una domanda semplice, quasi banale, secondo me non lo è, è tutt'altro che semplice, tutt'altro che banale. Personalmente ho passato gli ultimi tre anni tentando di indagarla ${ }^{36}$.

La stessa motivazione si ritrova nel romanzo, là dove Robledo pensa di aver trovato la chiave del suo reportage nelle giustificazioni delle persone che lavorano "per il gusto di lavorare":

Lo faccio perché mi piace, perché non posso fare altrimenti, perché prima o poi mi pagheranno, perché è un master, mica un lavoro, perché è un tirocinio, mica un lavoro, perché è un'opportunità che va colta al volo, perché non ho meglio da fare [...] Quante volte ho sentito questi discorsi? [...] Perché facciamo di tutto pur di non ammettere che lavoriamo solo perché ci piace? Possibile che sia stato io il primo a farmi queste domande? ${ }^{37}$

Mentre la studiosa Vincenza Pellegrino riconosce nella "cattura" la condizione "relazionale" del lavoro precario ${ }^{38}$, lo scrittore Zito si chiede se il lavoro non salariato non abbia una presa particolare sulla sua generazione che egli in un'intervista su La solitudine di un riporto aveva definito una "generazione di passaggio":

La generazione a cui appartengo e quella a cui appartengono i miei fratelli e le mie sorelle maggiori, sono generazioni di passaggio. Storicamente per generazioni di questo tipo non c’è alcuna speranza. La Storia però ha uno strano modo

36. Valentini David, “IntervistaaDanieleZito, comenasce Robledo?”, AltriAnimali,13giugno 2017, http:/ / www.altrianimali.it/2017/06/13/intervista-daniele-zito-nasce-robledo/

37. Ziтo Daniele, Robledo, cit., p. 51 (corsivo nell'originale).

38. Pellegrino, in un saggio sul lavoro precario all'università, ipotizza che "la 'cattura' oggi si caratterizzi proprio per le nuove e diverse condizioni relazionali del lavoro collaborativo che ricuciono la distanza tra precari e strutturati. Per contrastare la 'cattura', è necessario assumerne che essa dà risposta a bisogni relazionali che cerchiamo di soddisfare". Pellegrino Vincenza, "Lavoro cognitivo, passioni e precarietà. Per una 'resistenza relazionale' alle forme di cattura del sistema produttivo", in EAD. (a cura di), R/esistenze precarie. Lavoratori universitari e capitalismo cognitivo, Verona, Ombre corte, 2016, p. 51. 
di "far rima", non è detto che stavolta da tutto questo spaesamento non nasca qualcosa d'interessante. Al momento, però, non vedo nulla di incoraggiante ${ }^{39}$.

La condizione precaria non si limita quindi al precariato accademico ma è tipica delle categorie più vulnerabili, di età, genere e provenienza. Tra le "Testimonianze (quaderno V)" raccolte da Robledo per imbastire il suo reportage di "umanità", le età riportate vanno dai 60 anni - "Carlo M., 60 anni, siciliano" - ai 20 - "Ciro, 28 anni, campano"40 - con una concentrazione sui 30-40. Le storie di donne (due) costituiscono una minoranza ma sono anche tra le più tragiche perché parlano di umiliazioni e molestie sessuali sul lavoro". "Antonio C., 37 anni, pugliese" prima di unirsi a LPL era "un ricercatore universitario. Pardon, un ricercatore universitario precario", e definisce quelli come lui non "eroi", né "terroristi" ma "soltanto dei cretini" A2. Altri parlano della "rabbia" che li accomuna: "Ciro, 28 anni, campano" - "Io sono un ghost worker e così mio fratello. [...] Tu mi chiedi perché siamo entrati nell'organizzazione. Per rabbia, come tutti”"33.

Dice Zito in un'intervista di aver trovato il cognome del suo protagonista, Robledo, sull'elenco telefonico, mentre con il nome lo scrittore ha voluto ricordare il "Michele che si è suicidato e ha scritto una lettera al ministro Poletti", gesto che evidenzia il fatto che "di non lavoro si muore, e pure tanto, anche in Italia" ${ }^{4}$. La lettera, pubblicata per volontà dei genitori sul Messaggero Veneto del 7 febbraio 2017, contiene elementi che ritroviamo in forma romanzata nella scrittura di Robledo. L'idea del "percorso di liberazione", la soluzione estrema del suicidio scelta da chi si associa ai gruppi LPL, è già presente in nucleo in questa lettera che parla di "alto tradimento":

Lo stato generale delle cose per me è inaccettabile, non intendo più farmene carico e penso che sia giusto che ogni tanto qualcuno ricordi a tutti che siamo liberi, che esiste l'alternativa al soffrire: smettere. Se vivere non può essere un

39. PArisi Lucilla, “Un'intervista con Daniele Zito", Liberi di scrivere, 6 novembre 2013, https://liberidiscrivere.com/2013/11/06/un-intervista-con-daniele-zito-a-curadi-lucilla-parisi-2/.

40. Ziтo Daniele, Robledo, cit., p. 130, p. 137.

41. "Ed eccolo, l'ascensore sociale di cui tutti parlavano: mi premeva il culo, andava su e giù per la vagina, mi teneva ferma la testa, mi sussurrava all'orecchio che sono una troia, che se lo andavo a dire in giro mi avrebbe fatto ancora più male. Era quello l'ascensore sociale. Era quella lì, la nostra Repubblica fondata sul lavoro”, Ibid., p. 133.

42. Ibid., p. 135.

43. Ibid., p. 137.

44. Cirillo Jacopo, "Molte birre con... Daniele Zito", Finzioni Magazine, 19 settembre 2017, http:/ /www.finzionimagazine.it/f/molte-birre-con-daniele-zito/. 
piacere, allora non può nemmeno diventare un obbligo, e io l'ho dimostrato. Mi rendo conto di fare del male e di darvi un enorme dolore, ma la mia rabbia ormai è tale che se non faccio questo, finirà ancora peggio, e di altro odio non c'è davvero bisogno ${ }^{45}$.

La lettera si chiude con un p.s.: "Complimenti al ministro Poletti. Lui sì che ci valorizza a noi stronzi". Il ministro del lavoro, fautore del "decreto Poletti" che nel 2014 sanzionò l'entrata in vigore del Jobs Act, era già stato destinatario di un'altra lettera di una giovane arrabbiata, la ricercatrice di economia emigrata in Francia Marta Fana, che nel 2017 pubblica il pamphlet di grande successo Non è lavoro, è sfruttamento ${ }^{46}$. Nella lettera, pubblicata il 20 dicembre 2016 su L'Espresso, Fana aveva accusato il ministro che ha ideato i voucher di aver ridotto a un "gratta e vinci" l'opportunità che dovrebbero cogliere i giovani che non lasciano l'Italia, e ad aver aumentato la disuguaglianza sociale per chi resta:

Non è chiaro, Ministro Poletti, cosa sia per lei un'opportunità se non questa cosa qui che rasenta l'ignobile tentativo di rendere ognuno di noi sempre più ricattabile, senza diritti, senza voce, senza rappresentanza. Eppure la cosa che mi indigna di più è il pensiero che l'opportunità va data solo a chi ha le competenze e il saper fare ${ }^{47}$.

Claudio Panella invita a confrontare il romanzo di Zito con il saggio di Fana in una rassegna sull'Indice dei Libri del Mese in cui si sofferma su quelle opere di genere ibrido che, a partire dal G8 a Genova e a seguito di disastri nazionali come i morti sul lavoro della Thyssen Krupp o i malati di esposizione all'amianto di Monferrato, hanno iniziato a "raccontare le mutazioni del mondo del lavoro contemporaneo" e "testimoniano in maniera inequivocabile un fenomeno che nel dibattito letterario italiano si è voluto ascrivere alla crisi del paradigma postmodernista, identificando l'elaborazione di un canone nuovo a cui in molti si sono riferiti nei termini di un 'ritorno al reale' dei nostri scrittori'. Panella descrive il soggetto ambiguo di Robledo come il "lavoro che crea e divora

45. "La lettera di Michele che si è ucciso a trent'anni perché stanco del precariato e di una vita fatta di rifiuti", Messaggero Veneto, 7 febbraio 2017, https://messaggeroveneto.gelocal.it/udine/cronaca/2017/02/07/news/non-posso-passare-il-tempo -a-cercare-di-sopravvivere-1.14839837.

46. Fana Marta, Non è lavoro, è sfruttamento, Roma-Bari, Laterza, 2017. Sul Jobs Act si veda Fana Marta, Guarascio Dario, Cirillo Valeria, "Did Italy Need More Labour Flexibility? The Consequences of the Jobs Act", in Intereconomics, 2, 2016, pp. 79-86.

47. “La lettera di Marta al ministro Poletti", L'Espresso, 20 dicembre 2016, http:/ / gilioli. blogautore.espresso.repubblica.it/2016/12/20/la-lettera-di-marta-al-ministro-poletti/. 
il mondo", e "che crea e distrugge le nostre identità sociali ed esistenziali", e conclude che "il rovesciamento paradossale operato dall'autore siciliano è di quelli che fanno pensare e riavvolgere tutta la storia del lavoro tra xx e XXI secolo" $"$.

\section{CONGETTURE E DISTORSIONI DELLA VERITÀ}

Tra le testimonianze raccolte nel Quaderno V, quella dello scrittore di nicchia "Roberto, 42 anni, marchigiano" potrebbe funzionare come un cameo. A una delle presentazioni del suo ultimo libro L'elemento umano, Roberto si innamora di un lettore che gli chiede di scrivere come dedica "Lavoratore a oltranza". Lo scrittore dopo aver assistito al tragico "percorso di liberazione" dell'amico, diventa anche lui un ghost worker, "nel tentativo disperato di dare un senso a quella dedica da cui tutto era iniziato". Vuole essere "una sonda" e servire "al carotaggio" dato che scrivere "non serve". Solo così si rende conto che i tempi e gli uomini sono cambiati e ha "finalmente gli occhi per capirlo": "Andiamo tutti quanti nella stessa direzione $[. .$.$] verso una società dove lavoro e salario$ saranno una cosa completamente differente. Solo che non ce ne accorgiamo" ${ }^{\prime 4}$.

In un'intervista sul primo romanzo, il cui protagonista è un libraio "folle", la risposta di Zito alla giornalista sugli "eroi dei nostri tempi" contiene in nuce l'idea base di Robledo, l'indagine romanzata sui ghost workers, tanto che è possibile riconoscerne alcuni elementi nei brani sopra citati:

A occhio e croce, direi che il precario è una figura iconica di questi anni; anche i rivoluzionari senza rivoluzione, o meglio dentro un conflitto parcellizzato che non riesce mai a esprimersi in modalità condivise, costretti, loro malgrado, a un individualismo indotto, probabilmente lo sono [...]. Siamo dentro una mutazione profonda delle strutture che regolano la società e la sua compagine economica. Il libraio, a suo modo, esprime alcuni dei tratti caratteristici di tutte queste figure. Nel libro provo a descrivere una rabbia che è sia personale che collettiva; ciò consente al libraio di essere fratello sia del precario, che del ragazzo che sogna il riot, che dell'esodato. La vicenda del libraio tenta anche di descrivere un possibile arco esistenziale di figure come queste, proponendo anche una soluzione di fuga, diciamo così, abbastanza estrema. Non mi sembra che la realtà, nel nostro paese, stia evolvendo verso scelte di quel tipo, per cui,

48. Panella Claudio, "Realtà e surrealtà del lavoro in letteratura. Il Moloch che crea e divora il mondo", L'Indice dei Libri del Mese, 10 ottobre 2017, p. 13.

49. Ziтo Daniele, Robledo, cit., p. 139, pp. 141-143. 
ad oggi, mi sa che il mio libraio più che essere un eroe dei nostri tempi, sia al contrario un antieroe ${ }^{50}$.

Che i precari siano dei "rivoluzionari senza rivoluzione" dentro un "conflitto parcellizzato" è un'idea che si ritrova in diversi studi che cercano di teorizzare la "resistenza" del precariato su nuove basi, in cui l'Italia figura come un "laboratorio" 51 . Nel capitolo "Leggende metropolitane (quaderno VIII)" si trova l'inventario steso da Robledo in un file intitolato "Dicevano di loro". In esso si ritrovano diverse teorie sul movimento del Lavoro per il Lavoro da punti di vista "plausibili" ma sempre distorti dalla finzione. Una di queste è l'opinione molto diffusa secondo cui "il movimento del Lavoro per il Lavoro sia pagato dalle multinazionali per creare quelli che, con un termine un po' troppo violento, chiamano 'gli schiavi $2.0^{52}$ [...] Stando alle parole dei fautori di tale ipotesi, l'Italia è stata scelta come laboratorio politico di questo nuovo tipo d'esperienza"53. Un'altra è la (finta) "visione pessimistica" di Zygmunt Bauman che parla di "Rivoluzione liquida" per indicare "rivolte interne al sistema produttivo" a cui "non segue alcuna formalizzazione di nuovi rapporti di potere, ma soltanto la morte" 54 .

Come indica giustamente Isidoro Meli su la Repubblica, il romanzo, oltre alla tematica del precariato lavorativo ed esistenziale, tocca un altro tema attuale che è quello della "natura sfaccettata della realtà, l'impossibilità di pervenire a una verità univoca, piatta, risolutiva" 55 . Se Panella si rifà al G8 per segnare lo spartiacque tra postmoderno e "ritorno al reale" 56 , Zito, che oltre a essere ricercatore

50. PARIsi Lucilla, "Un'intervista con Daniele Zito", cit.

51. Si veda p.es. il manifesto recente di Foti Alex, Theory on Demand \#25. General Theory of the Precariat, Institute of Network Cultures, Amsterdam, 2017, in cui si difende l'ipotesi che il precariato, essendo la classe più sfruttata, sia anche quella che crea il maggior valore sociale, come hanno dimostrato le diverse rivoluzioni e insurrezioni del 2011 (p. 5). La genesi del movimento antiglobalista EuroMayDay negli anni 2001-2008 viene riportata all'Italia (p. 16).

52. Qui potrebbe trattarsi di un riferimento al libro manifesto di GriLLo Beppe, Schiavi moderni. Il precario nell'Italia delle meraviglie, Milano, Casaleggio Associati, 2007.

53. Ziтo Daniele, Robledo, cit., p. 210.

54. Ibid., p. 212 e p. 211.

55. MeLi Isidoro, "Robledo di Zito il paradosso del precariato", la Repubblica, 1 luglio 2017, https://ricerca.repubblica.it/repubblica/archivio/repubblica/2017/07/01/ robledo-di-zito-il-paradosso-del-precariatoPalermo10.html.

56. Panella si riferisce a Donnarumma Raffaele, Policastro Gilda e Taviani Giovanna, "Ritorno alla realtà? Narrativa e cinema alla fine del postmoderno", in Allegoria, XIX, 57, 2008, pp. 7-95. 
ha frequentato attivamente dei centri sociali ${ }^{57}$, prende come esempio la sua esperienza personale al G8 di Genova per dimostrare come uno scontro di questo tipo in pratica sia inenarrabile, se non partendo proprio dalla documentazione necessariamente parziale dell'evento:

Ma il nostro rapporto con la verità è mediato dalle lotte tra $\mathrm{i}$ vari revisionismi di quella verità. Sedici anni fa, ho partecipato al G8 di Genova; se uno dovesse farsi un'idea di quel G8 a partire da tutti i documenti e le interviste degli anni successivi, si farebbe un'idea costruita su più distorsioni della realtà, quella degli attivisti, dei black bloc, della polizia, dei media. La verità è un processo complesso che deriva dalla somma delle sue distorsioni. E il mio libro funziona cosi $i^{58}$.

Di questa tecnica di "distorsione" testimoniano il già citato Quaderno VIII, "Leggende metropolitane" e anche il Quaderno VII, "Interviste", che raccoglie il materiale che Robledo ha preso dal web, tra cui conferenze stampa con le autorità che dimostrano chiare somiglianze con quelle seguite ai "fatti di Genova" ${ }^{59}$. Dice Zito di aver voluto creare una "bufala" che, oltre a trarre ispirazione dai reportage sui morti sul lavoro su cui si è documentato per scrivere il romanzo, si nutre delle sue conoscenze sui movimenti rivoluzionari degli anni Sessanta e Settanta tramutatisi in gruppi terroristici:

La matrice terroristica deriva dalla mia esigenza di inserire anche i morti sul lavoro. Queste persone diventano statistica solo quando hanno un contratto, se non ce l'hanno non esistono. Volevo dare un senso a queste morti, creare una bufala che le integrasse ${ }^{60}$.

Interessa all'autore, come spiega in un'intervista con Rai Scuola, proprio questo meccanismo per cui un malessere e degli interessi condivisi, che creano collettività, possano mutarsi in un'ideologia che legittimi la violenza, come

57. "Io vengo da un'esperienza molto lunga dentro un centro sociale a Catania e ho visto tantissime conferenze stampa in cui i prefetti tentavano di raccontare versioni diverse dalla realtà dei fatti. E introdurre quel linguaggio e quell'ambiguità nel libro mi divertiva"; Cirillo Jacopo, "Molte birre con... Daniele Zito", cit.

58. Ibid.

59. Alla "Conferenza stampa convocata dal Ministro dell'Interno in seguito ai fatti del 22 novembre 2013", alla domanda del giornalista "Gli agenti hanno fatto fuoco su di loro, senza il minimo preavviso [...] I commentatori di tutto il mondo parlano di macelleria messicana", così risponde il ministro: "La invito a moderare i toni. Le ricordo che è in presenza di una delle più alte cariche dello Stato" (Zito Daniele, Robledo, cit., pp. 198-99). Corsivo nell'originale.

60. Cirillo Jacopo, "Molte birre con... Daniele Zito", cit. 
accade nel romanzo con i gruppi LPL che da una giusta causa si trasformano in un'organizzazione terroristica con un'idea malata di "purezza" del lavoro ${ }^{61}$. Questo tema è anche al centro dell'opera sperimentale che Zito scrive tra il 2016 e 2017 e pubblica nel 2019, Uno di not ${ }^{62}$, in cui, sul modello della tragedia greca, l'autore, con la stessa scrittura corale di Robledo, cerca di ricostruire la mentalità che permette il dilagare dell'ideologia del postfascismo e che legittima le persone a compiere atti di violenza in un clima di impunità ${ }^{63}$.

È lo scrittore Claro, nel suo "feuilleton littéraire" su Le Monde, a evidenziare ai lettori francesi l'originalità dell'approccio narrativo con cui Zito riesce a immaginare la realtà del "piacere di lavorare" con un romanzo:

Le travail, c'est la santé sociale - plutôt que morale, mentale ou physiologique. Pour mettre à nu ce degré de cynisme, que peut la littérature ? L'écrivain Daniele Zito n'a pas à pousser les curseurs très loin pour imaginer le pire. Le pire était déjà là, larvé, en attente. Il l'a laissé prospérer et son roman Robledo en est la preuve glaçante ${ }^{64}$.

Sulla rivista belga PointCulture il romanzo viene inserito in un'indagine di Benoit Deuxant sulle finzioni speculative che vertono sul lavoro e così viene accostato a classici come 1984 di Orwell e Brave New World di Aldous Huxley. Risulta da questo studio comparativo che sia l'assoggettamento al lavoro che la questione della sua scomparsa sono al centro di opere di fantascienza costruite come finzioni distopiche in cui basta, come nel caso di Zito, "pousser une hypothèse dans ses limites les plus extrêmes pour révéler, comme dans une fable ou une parabole, des traits insoupçonnés non pas d'un futur imaginaire mais bien de notre société actuelle"65.

61. "Dalla trentesima edizione del Salone del Libro di Torino: Daniele Zito e i suoi lavoratori fantasma", Rai Scuola, http://www.raiscuola.rai.it/articoli/daniele-zito-e-i-suoi-lavoratori-fantasma/37335/default.aspx.

62. Ziтo Daniele, Uno di noi, Torino, Miraggi edizioni, 2019.

63. "[L]'unico modo per descrivere realmente il contesto [è] utilizzare diversi punti focali [...]. Come in Robledo, il mio precedente romanzo, per buona parte del libro la storia emerge dalla contrapposizione di questi punti di vista"; FAzIO Salvatore Massimo, "Daniele Zito: 'Nessuno è innocente", Vivere. Allegato de La Sicilia, 19 settembre 2019, http://www.viveregiovani.it/news/libri/283132/daniele-zitonessuno-e-innocente.html.

64. Claro, "La 'tâche' aveugle", Le Monde, 5 aprile 2019.

65. Deuxant Benoit, "Le futur du travail. Survol des fictions spéculatives sur le travail", PointCulture, 24 ottobre 2018, https://www.pointculture.be/magazine/articles/ focus/le-futur-du-travail/. 
Un lettore al circolo di Ivrea chiede all'autore se con il reddito di base non si entri in un nuovo paradigma ${ }^{66}$. La domanda viene affrontata da Zito in un contributo sul reddito di cittadinanza apparso nella Newsletter cheFare in cui mette in discussione i vantaggi del workfare - "work for welfare, lavorare per avere un beneficio" 67 - avvisando che i vincoli neoliberali rischiano di violare la "libertà dei singoli" e potrebbero trasformare la misura "di sinistra" in un "dispositivo disciplinare totalizzante" ${ }^{\prime 68}$. Sulla stessa Newsletter Zito si misura anche con la questione della temporanea restrizione dei diritti esperita da migranti legali e illegali, dato che tali diritti sono concepiti in base a un criterio di appartenenza nazionale che prende "la stanzialità come la norma", mentre è prevedibile che i flussi migratori spostino la cittadinanza verso la normatività di una "mobilità" transnazionale. Pronostica Zito: "Nasceranno meccanismi per dare maggiore peso politico alla mobilità (al movimento, al flusso) e non più alla nascita, come avviene oggi. Migranti e autoctoni avranno diritti meno sbilanciati di quelli odierni. Ciò inevitabilmente comporterà delle tensioni più o meno cruente" ${ }^{\prime 6}$.

Adottando la struttura della pseudo inchiesta Zito ha offerto, in forma di finzione, una lucida disanima della precarietà economica ed esistenziale, immaginando le liberazioni nelle loro forme più estreme messe in atto dai gruppi $\mathrm{LPL}^{70}$, e con una particolare attenzione per "l'elemento umano", per riprendere il titolo di Roberto, scrittore e ghost worker. I nuovi paradigmi richiedono che la realtà continui a essere investigata, "attraverso la falsificazione" ${ }^{71}$ e derivando la verità "dalla somma delle sue distorsioni"”2.

Monica Jansen Utrecht University

66. Cirillo Jacopo, "Molte birre con... Daniele Zito", cit.

67.ZITо Daniele, "Cosa sarà il reddito di cittadinanza. Dal welfare al workfare", cheFare, 5 ottobre 2018, https://www.che-fare.com/reddito-cittadinanza-welfare-workfare/.

68. Ibid.

69. Ziтo Daniele, "Le logiche della demografia e quelle dell'appartenenza", cheFare, 21 novembre 2018, https://www.che-fare.com/logiche-demografia-appartenenzamigrazioni/.

70. In epigrafe di Robledo, Zito riporta la scritta posta sopra il cancello d'ingresso di Auschwitz, "Il lavoro rende liberi".

71. Ibid., p. 256.

72. Cirillo Jacopo, "Molte birre con... Daniele Zito", cit. 